\title{
Acknowledgment of Ad Hoc Reviewers
}

The editors acknowledge the generous and valuable help from the following reviewers who, in addition to the Editorial Board members, reviewed manuscripts submitted for publication on an ad hoc basis for the 2016 volume.

\begin{tabular}{|c|c|}
\hline Tahirah Abdullah & Mark E. Courtney \\
\hline Becci Akin & Rebecca Cox \\
\hline Debby Allen & E. Mark Cummings \\
\hline Michaelle Amering & Cheryl Currie \\
\hline Pamela Anderson & Lisa Curtin \\
\hline \multicolumn{2}{|l|}{ Scott Anderson } \\
\hline Sandra Annan & Danielle Dallaire \\
\hline Toni Antonucci & Steven J. Danish \\
\hline Tichianaa Armah & Graham Danzer \\
\hline Deanne Armstrong & Carl D’Arcy \\
\hline Laura Armstrong & Carolyn Joy Dayton \\
\hline Thalida Arpawong & Scott Decker \\
\hline Shervin Assari & Diane DePanfilis \\
\hline \multirow[t]{2}{*}{ Majdouline Aziz } & Daniel Dodgen \\
\hline & Mary Dozier \\
\hline Yaacov Bachner & Christopher F. Dresche \\
\hline Venera Balidemaj & Amy Dworsky \\
\hline \multicolumn{2}{|l|}{ Kira Hudson Banks } \\
\hline Jim Banta & M. Ann Easterbrooks \\
\hline Armando Barragán & Nicholas Eaton \\
\hline Michelle Greenspoon Barrett & Christopher Eckhardt \\
\hline Deborah Belle & Dikla Eckshtain \\
\hline Rezarta Bilali & Marina Economou \\
\hline Zeynep Biringen & Katherine Ehrlich \\
\hline Gary Blau & Christopher Erbes \\
\hline Jessica Borelli & Michelle Evans-Chase \\
\hline \multicolumn{2}{|l|}{ Christopher Bory } \\
\hline Jennifer E. Boyd & Melissa Faith \\
\hline Daniel Bradford & Kostas A. Fanti \\
\hline Jonathan Bradshaw & Jamison Fargo \\
\hline Lauren Brinkley-Rubinstein & Erika Felix \\
\hline Elizabeth Bromley & Olga Maria Fernandez \\
\hline Timothy Brown & Adam Fingerhut \\
\hline Ann Buchanan & Jeremy Finn \\
\hline Eli Buchbinder & Matia Finn-Stevenson \\
\hline Stephanie Budge & Sheehan Fisher \\
\hline Daniel Burland & Kevin Fitzpatrick \\
\hline Barbara Jean Burns & Julian Ford \\
\hline Amy C. Butler & Catherine Forde \\
\hline Susan Cadell & Thomas Gabriel \\
\hline Arnie Cann & Larry Gant \\
\hline Etzel Cardeña & Edward F. Garrido \\
\hline Deven Carlson & Elizabeth Gassin \\
\hline Tony Cassidy & Maurice Gattis \\
\hline Kathryn Ann Castle & Brenda Geiger \\
\hline Asa Cater & Sheri Gibson \\
\hline Yvonne Chase & Kathleen Gilbert \\
\hline Jill Chonody & Virginia Gil-Rivas \\
\hline Krista Chronister & Sari Gold \\
\hline Paula Cloutier & Seana Golder \\
\hline Kendell Lamonte Coker & Joseph Gone \\
\hline Cyleste Collins & Jessica R. Goodkind \\
\hline Melissa Lynne Morgan & Jessica Rose Graham \\
\hline Consoli & Josefina Grau \\
\hline Anne Conway & Matt Gray \\
\hline Shauna Cooper & Sarah Gray \\
\hline Katie L. Cotter & Elizabeth Greeno \\
\hline
\end{tabular}

Robert Griffore

Gertrud Sofie Hafstad

Scott Hanson-Easey

William English Hartmann

Kirsten Havig

Traci Hefner

Claire Henderson

Susan Henney

Rachel Hershberg

Devon Hinton

Stevan Hobfoll

Sharon Hodges

Steven Hoffman

Melissa Holtzman

E. Scott Huebner

Jaclyn White Hughto

Marisha Humphries

Rachel Zack Ishikawa

Kelly Jackson

James Jaranson

Shabnam Javdani

Eranda Jayawickreme

Andrew B. Jeon

Waldo Johnson

Pooran C. Joshi

Femmie Juffer

Zornitsa Kalibatseva

Hugo Kamya

Evangelos Karademas

Carmit Katz

Joy S. Kaufman

Traci M. Kennedy

Vivian Khamis

Siamak Khodarahimi

Maryam Kia-Keating

Sean Kidd

Cara Kiff

Jin E. Kim

Mimi Kim

Joe Kosciw

Derrick Alan Kranke

Terry Krupa

Sharon F. Lambert

Paul Lanier

Osnat Lavenda

Iris Lavi

Justin Lavner

Christopher Layne

Lucy Le Mare

Scott C. Leon

Amber Letcher

Brigitte Leuger-Schuster
Terri Lewis

Junlei Li

Stephanie Lichtenfeld

Jae Lim

Sarah Ketchen Lipson

Daniel Losen

Ellen Lukens

Andrew Macnab

Sandy Magaña

Kathryn Magruder

Ron Manderscheid

Anthony Marcus

Carolina Marin

Alexa Martin-Storey

Tyler Mason

Jason Matejkowski

Jennifer Matjasko

Meredith Matone

Carolyn McCarty

Lucy Sarah McGregor

Thomas J. McMahon

Paul Miller

Dalen Monica

Eduardo S. Morales

Tally Moses

David Paul Moxley

Pietro Muratori

Yochay Nadan

Julie L. Nagoshi

Teresa Nguyen

Randy Noblitt

Lydia P. Ogden

Yin Paradies

Sita G. Patel

Desmond Patton

Einat Peled

Brea Perry

Amanda Phelan

Alex Pieterse

Ronald Pitner

Seth Prins

David Pyrooz

Athena Ramos

Lisa Razzano

Gwyther Rees

Daniel Rempala

Harmony R. Rhoades

Eric M. Rodriguez

Ami Rokach

Jessica Rosenberg

Rosa Rosnati

Abigail Ross

Mary Jane Rotheram-Borus 
Sonia Rubens

Glenda Russell

Benedetto Saraceno

John Sargent

Nina Sayer

Jamie A Seabrook

Mary V. Seeman

Uma Segal

Heather Pane Seifert

Gianluca Serafini

Chad Shenk

Darcy Clay Siebert

Derrick Silove

Russell Skiba

Sondra Smith-Adcock
Cathy Sowell

Wesley Eugene Sowers

Julie Spielberger

Victoria Stanhope

Cesalie Stepney

Bradley Stolbach

Roni Strier

Eric Swank

James A Swartz

Susan Swearer

Elayne M. Tanner

Brian Taylor

Laura Taylor

Martie Thompson

Luanne Thorndyke
Christopher J. Trentacosta Jack Tsai

Carolyn Tubbs

Anaise Tuepker

Jennifer Urban

Alice P. Villatoro

Shelley MacDermid Wadsworth

Geoffrey R. Waghorn

David Wang

Weijun Wang

Zhenlin Wang

Earlise Ward
Martin Wasserberg

Wayne Wilkinson

Monnica T. Williams

Joshua Mosquera Wilson

Melvin Wilson

Douglas Wolf

Philip Yanos

Ekaterina Yazykova

Patrick Zapata

Charles Zeanah

Karen Zilberstein

Irene Nathan Zipper

Alyssa Zucker 